\title{
Method for heterogeneous sample analysis on LCMS without sample manipulation
}

\author{
Muhammad Alimuddin*, Brandon Phung, William Kim, Paul Richardson, Wei Wang
}

Pfizer-La Jolla, 10770 Science Center Drive, San Diego, California, 92121

\begin{abstract}
We describe a simple, effective, and automated method that tolerates heterogeneous sample analysis on liquid chromatography mass spectrometer (LCMS) systems without any sample manipulation. This was developed to mitigate the inconsistent sample preparations that often arise when LCMS systems are operated in a self-service mode (walkup or open access), such as in a pharmaceutical research environment. We demonstrated the robustness of our method through 144 sequential injections of a heterogeneous sample on the LCMS without observing clogging or an increase in the system pressure, whereas with a conventional set-up, the same sample resulted in an almost instantaneous clog. Analysis of carefully tracked metrics for six walkup LCMS over 3 years since the rollout of this method at our facility has shown $>95 \%$ sustained reduction in clogging rate, accompanied by over a 30 -fold increase in operational robustness, all with no impact on the quality of the chromatography. Implementation has further lead to savings in time and resources for both the system administrators and users, hence making walk-up LCMS easier to use yet harder to break, with the additional benefit of providing data for heterogeneous samples available to the user, and reducing sample carry-over. The method is inexpensive to implement and requires neither expensive hardware such as pumps or valves, nor any changes to existing LCMS methods.
\end{abstract}

Keywords: heterogeneous sampling, reversed phase high performance liquid chromatography, injection, clogging, walk-up, open access, LCMS, reaction monitoring

* Corresponding author: Tel.: +1 858395 7074; fax: +1 8586788164 .

E-mail address: muhammad.alimuddin@pfizer.com

\section{Introduction}

In a fast-paced pharmaceutical research environment, the operation of liquid chromatography mass spectrometry (LCMS) instruments in a "walk-up" or "open-access" selfservice mode is well-established [1-3]. The LCMS continues to be the pivotal tool for not only monitoring chemical reactions, but also assessing the purity and/or integrity of the resulting products. Robust modern instrumentation aligned with an easy to use software interface enables rapid uptake from a diverse user group. Currently here in Pfizer, La Jolla, we operate six LCMS systems in this fashion for $c a .35$ chemists running over 30000 samples a year with one expert analytical chemist assigned to monitor (calibration, response to errors, routine repairs etc.) these instruments. In this environment, the biggest variable in LCMS systems arises from numerous users assessing a wide array of different chemistries. Despite the users' attentive efforts towards sample preparation for analysis, often the sample's inherent physical and chemical properties lead to the major cause of instrument failure being clogging, which leads to the subsequent overpressurization and shutdown of the system. This problem has long been recognized and despite its 
apparent simplicity, it continues to plague this analytical technique particularly in a walk-up environment. Examination of our instrument performance metrics, which we have tracked since 2010 , revealed that typically in a single year, $54 \%$ of our service calls to remediate instrument shutdown were directly linked to clogging by heterogeneous samples (Table $1 \&$ Supplmental Information, SI). This leads to several knock-on impacts for the administrator and users alike including; 1) reduced instrument capacity for users due to instrument down-time, 2) resource requirements for the administrator to troubleshoot, repair, and maintain the performance of the instrument, 3) potential cost of parts to repair the instrument if the component in which the source of the blockage is located cannot be cleaned out, and 4) pre-blockage, unreliable chromatography due to pressure spikes as debris builds up accompanied by sample carry-over leading to chemists potentially having to repeat LCMS experiments, or being unable to make decisions based on the analytical data obtained

Table 1. Breakdown on the causes of failure for walk-up LCMS instruments in 2014

\begin{tabular}{|c|c|c|c|}
\hline Cause of Failure & $\%$ & Location of Problem & $\%$ \\
\hline \multirow{6}{*}{ Instrument } & \multirow{6}{*}{41} & Software & 44 \\
\hline & & LC-Pump & 10 \\
\hline & & Sampler & 20 \\
\hline & & Column Heater & 4 \\
\hline & & UV Detector & 7 \\
\hline & & MS Detector & 15 \\
\hline \multirow{3}{*}{ Sample Clog } & \multirow{3}{*}{$54 *$} & Clog Location & $\%$ Clog \\
\hline & & Sampler to Column & 95 \\
\hline & & Column to MS Detector & 5 \\
\hline
\end{tabular}

*Note - The remaining 5\% balance of failures were due to logistic issues such as wrongly positioned or missing samples in the loading tray.

Despite this problem long having been recognized by the hardware manufacturers, incorporation of a needle-washing contingency prior to injection has proved only to be truly effective in terms of preventing cross-contamination rather than clogging. Several other techniques have been proposed to alleviate this critical problem, such as placing additional efforts on sample preparation or incorporating additional hardware such as a pump and valve to back-flush the LC [4]. A number of techniques exploit solid-phase extraction, in-line filtration/back-flushing or a combination thereof [5]. A number of these approaches require the purchase of an additional pump as well as changes to the existing set-up such as re-configuration of the valves to enable back-flush [6-7]. An alternative method to decrease the rate of clogging involves modification of the gradients used to increase the solubility of samples, which are not typically soluble in aqueous-based systems [8], though this approach creates significant additional work for the analytical chemistry team with the need to create custom chromatography conditions for each project based on the diversity of the chemical substrates under investigation,. Increasing the sample and column temperature has also been utilized to increase solubility, but 
this again is not a universal solution particularly as compounds can still crash when they encounter "cold spots" within the system, and in some cases the elevated temperatures the sample encounters can lead to compound decomposition [9]. Given that none of the solutions considered so far have been either universal or cost-effective, we report here our progress towards the design, validation, and implementation of a simple, inexpensive and automated method for heterogeneous sample analysis on LCMS whilst negating the need for extensive manipulation of the sample prior to analysis.

\section{Experimental}

\subsection{Materials and supplies}

Commercially available LCMS grade acetonitrile was purchased from Fisher Scientific (Fair Lawn, NJ, USA). Ammonium formate and formic acid were purchased from Aldrich (Milwaukee, WI, USA). HPLC grade $18.2 \mathrm{M} \Omega$ water was produce using a Milli-Q water purification system (Millipore, Bedford, MA, USA). Pfizer proprietary compounds were used for this study.

\subsection{Walk-up LCMS system}

All data was collected utilizing an Agilent 1260 LC with MSD Agilent model 6120 single quadrupole mass spectrometer detector. The LC binary pump (model G1312B) attached to an autosampler (model G1329B), which uses an external tray for sample submission. The column compartment (model G1316C), is attached to a diode array (model G1315C). Agilent Easy Access (rev. A.06) is used for sample submission and ChemStation (rev. B.05.01) software is used for acquisition and data handling. General OA methods consist of either a 2-or 3-minute gradient of 5 - $95 \%$ B over 1.5 or 2.5 minutes respectively, with a 0.5 minute hold at this solvent concentration. Solvent A: Water $(0.1 \%$ formic acid $+0.05 \%$ ammonium formate). Solvent B: Acetonitrile (5\% $\mathrm{H}_{2} \mathrm{O}+0.1 \%$ formic acid $+0.05 \%$ ammonium formate). The flow rate is $1.0 \mathrm{~mL} / \mathrm{min}$ using a Luna Omega Polar C18 column $(2.1 \times 30 \mathrm{~mm}, 1.6 \mu \mathrm{m}$ particle size $)$ maintained at a temperature of 60 ${ }^{\circ} \mathrm{C}$

\subsection{Heterogeneous sample analysis method}

To potentially resolve the issues of sample clogging, it is important to identify the most frequent clogging location within the LC system. Analysis of our service records indicated that approximately $95 \%$ of the clogs occurred between the needle seat and the column head (Table 1). To better understand why this region is so prone to accumulation of solid debris and subsequent clogging, it is instructive to examine Figure 1a, from which we can see as would be expected that the initial point of entry of solids into the system is from the needle seat. Hence, if we can eliminate particulate build-up at this specific position, then it stands to reason that we can reduce the instances of clogging.

With the need to effectively prevent solid particulate matter entering the system, a $0.2 \mu \mathrm{m}$ filter was placed inline after the needle seat. It was first envisioned that this filter would allow clean sample to pass through while retaining any solid debris from the sample as depicted in Figure 1b. However, gradual build-up of solid debris over a number of injections still lead to clogging. 
To remove the solid debris almost immediately after it has been deposited, this newly installed filter was back-flushed by diverting the flow of the mobile phase from the column (350 bar) to the filter (20 bar) using a T-connector (Valco part ZT1C) (Figure 1c). This simple technique of diverting the flow through a low pressure filter line taking advantage of the high back pressure of the column by adding a T-connector between these two lines has not to the best of our knowledge been previously reported. The automated back-flushing of the filter continues for approximately one minute after each injection thus preventing both debris build-up and sample carry over.

(a)

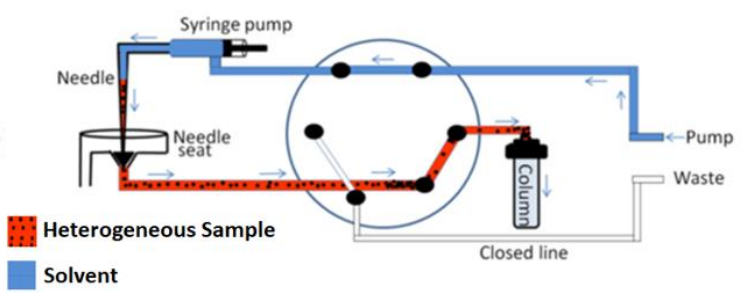

(b)

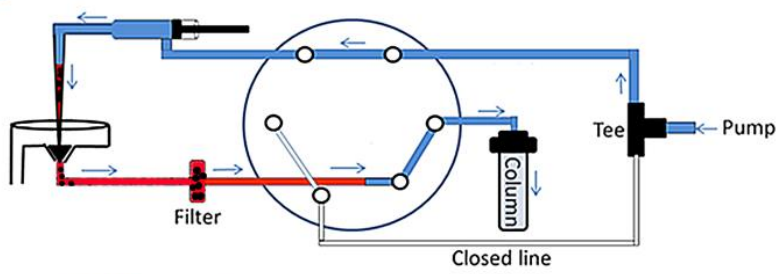

(c)

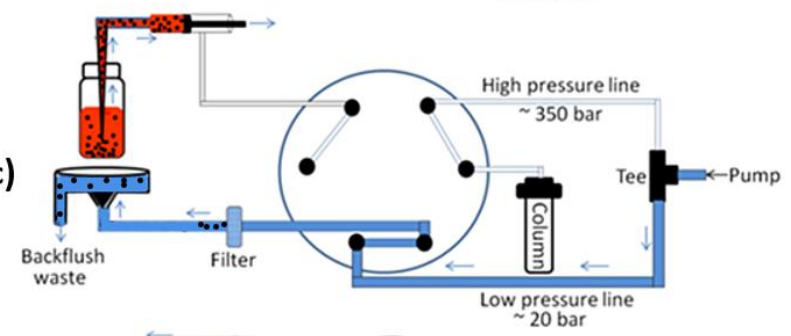

(d)

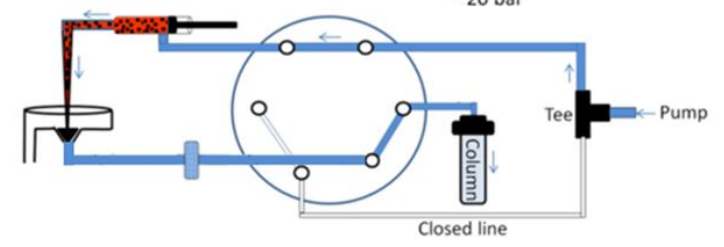

Figure 1: Heterogeneous sample analysis: Method filters and removes sample debris after each injection. (a) Flow path of a conventional sampler; (b) Filter and T-connector added to enable back-flushing; (c) Back-flushing removes sample debris; (d) Sampler is cleaned, and ready for next injection

\section{Results \& Discussion}

\subsection{Robustness of heterogeneous sample analysis method}

With the intention of alleviating multiple problems arising from instrument clogs, a novel though inexpensive plumbing set-up was implemented on our walk-up LCMS systems. The inherent novelty of our approach lies in the simplicity of the modification, not requiring an additional pump and valve, or any changes to the existing LC methods. This solution proved to be both simple and effective. We hypothesized that, by simple installation of a filter in tandem with serial back-flushing of the needle seat after each injection, we can drastically reduce the instances 
of clogging. To test the robustness of this method, a heterogeneous sample, which had previously been observed to instantly cause a shutdown due to a blockage on our standard instruments, was set to run sequentially on a modified LCMS system. This sample was observed to run for 144 consecutive injections over a period of 24 hours with no system shutdown, pressure errors or observation of pressure spikes providing a reasonable peak area reproducibility of $5.6 \%$ RSD (Figure 2) as compared to $1.7 \%$ for homogeneous samples (see Supporting Information). The method enables previously inaccessible LCMS data to be generated for heterogeneous samples.

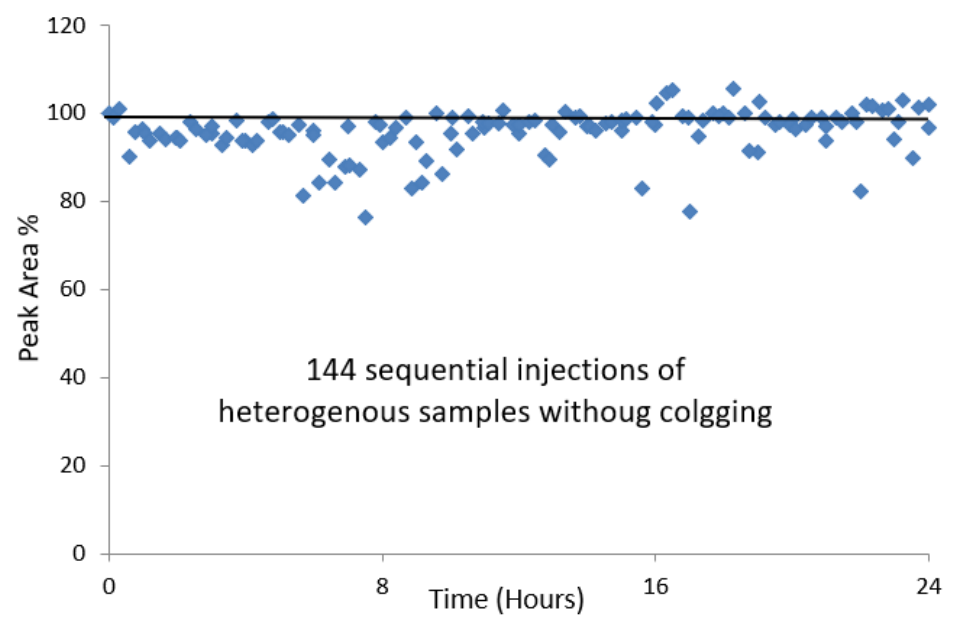

Figure 2: Demonstrating the robustness of heterogeneous sample analysis. No LCMS clogging observed after 144 sequential injections of a heterogeneous sample over a 24 hour period.

There are several other factors that need to be considered when implementing a process change in one's operation. One such factor is evaluating whether the change will have any significant impact on the chromatography. Given the set-up and positioning of the back-flush, we expected it to have a negligible effect on the chromatogram and this hypothesis is evidenced by the data presented in Figure 3, which shows a chromatogram before and after back-flush installation. The minor change in the peak retention time does not influence data interpretation as a mass spectrometer is attached to these systems for peak identification purposes. The overall modification of the system represents a significant gain in robustness for the walk-up instrument.

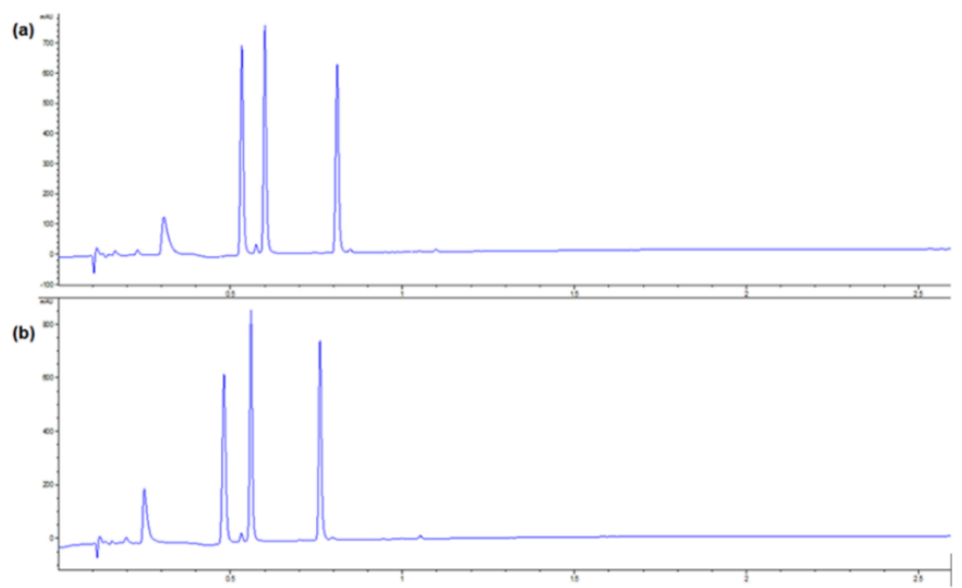

Figure 3: No significant change in chromatographic performance after the installation of the inline filter, and back-flush. (a) Sample prior to installation.(b) Sample post installation. 
Prior to deployment of the heterogeneous sample analysis method on all the walk-up LCMS instruments throughout the department, one final challenge remained to be surmounted. On most of our walk-up production LCMS injector systems (Agilent Model G1329B), there is no flush port for the controlled disposal of the solvent resulting from the back-flush process. As such, excess solvent from the back-flush is likely to congregate on the needle seat and shut down the instrument by triggering a leak alarm. To address this, a series of $1 \mathrm{~mm}$ holes were drilled in the needle seat cover allowing the solvent to drain through a custom-made drip tray into a suitable hazardous waste container for disposal (See SI Fig S-3).

\subsection{Performance of heterogeneous sample analysis method in medicinal chemisty}

With the design of our prototype system validated, we installed the heterogeneous sample analysis method on all our walk-up LCMS instruments. This proved to be the critical phase of this project, as these systems are closely monitored for performance metrics with comprehensive records compiled featuring details on time and reason for an instrument being out of service. Instrument performance has been constantly under evaluation since 2010, and Figure 4 provides a snapshot of the number of instances of "instrument clogging" over a four year period, with the back-flush being implemented and rolled out to the six walk-up LCMS sequentially over a 10 month period. We felt that this provides a representative body of data to provide an assessment of the impact of the change to our operations. It is important to stress that over this period, the number (and identity) of the users and the types of chemistries evaluated (typical medicinal chemistry reactions) [10] has remained approximately constant, while the number of samples run has increased. None of these instruments have either been replaced or undergone significant upgrades in the time of review, and each instrument typically hosts 6-8 users depending on its specific location within the building. The one exception herein is the placement of one of the instruments in a controlled high potency laboratory, which is used on a far less frequent basis than our other systems (vide infra). Given this, there were no extraordinary events (such as taking instruments out of commission either completely or for an extended period of time during the evaluation) at the time of the heterogeneous sample analysis method implementation shown in Figure 4 that could have also had such a sizable impact on the trends observed.

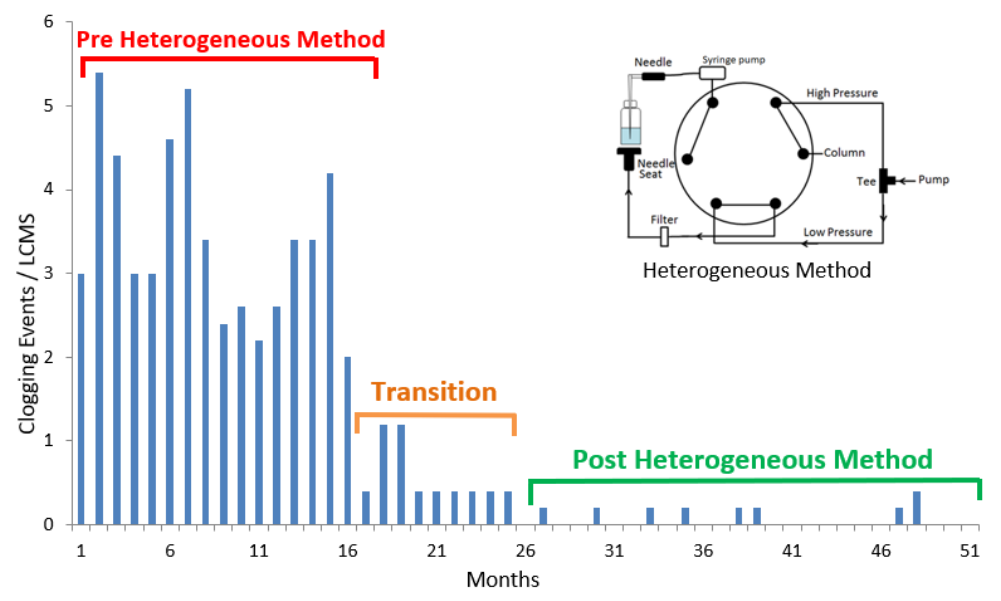

Figure 4: Impact of the heterogeneous sample analysis method on operations over a four year period. 
As Figure 4 illustrates, the implementation of the heterogeneous sample analysis method on walk-up LCMS has decreased the instances of clogging, naturally resulting in increased instrument availability and users' satisfaction. This method led to savings in time resource by allowing chemists to continue their work without interruption. Additionally, this has led to a significant savings in resources for our Analytical team, therefore allowing these chemists to focus on value-added and project-based activities. To assess the impact on an instrument by instrument basis, Table 2 provides details on the number of samples run across a 50 month period on each system (Instruments nominally labelled 1-6), as well as the number of clogging events per month prior to and after the implementation of the heterogeneous sample analysis method.

Table 2. Impact of heterogeneous sample analysis method on individual walk-up LCMS

\begin{tabular}{cccc}
\hline System & $\begin{array}{c}\text { Samples } \\
\text { over 50 } \\
\text { months }\end{array}$ & $\begin{array}{c}\text { Clogs/month } \\
\text { Pre } \\
\text { Implementation }\end{array}$ & $\begin{array}{c}\text { Clogs/month } \\
\text { Post } \\
\text { Implementation }\end{array}$ \\
\cline { 2 - 4 } Instrument 1 & 24189 & 4.25 & 0.08 \\
Instrument 2 & 17385 & 1.75 & 0.08 \\
Instrument 3 & 32822 & 5.25 & 0.17 \\
Instrument 4 & 33168 & 3.33 & 0.08 \\
Instrument 5 & 34814 & 2.83 & 0.04 \\
** Note - Instrument 6* & 2897 & 0.42 & 0 \\
\cline { 2 - 4 } Totals & 145275 & 17.83 & 0.46 \\
\hline
\end{tabular}

Figure 5 demonstrates that in 2014 (the last full calendar year without implementation of the heterogeneous sample analysis method on any instrument), 54\% of the service calls (of which there were in excess of 208) were to address clogging issues. This number was truly staggering and represented a resource drain on the Analytical team. For 2015, which represents a transitional year based on the fact that all the instruments were converted to incorporate the heterogeneous sample analysis method during this time (in total the instruments spent $\sim 40 \%$ of the year without back-flush), we saw 89 clogging events, which was a reduction of 120. For 2016, the first year that all six instruments had the heterogeneous sample method implemented, we observed a precipitous drop in clogging events leading to a steady state situation of approximately six clogs per year since, this represents a $97 \%$ reduction in clogging rate since we modified our operations to incorporate the new methodology.

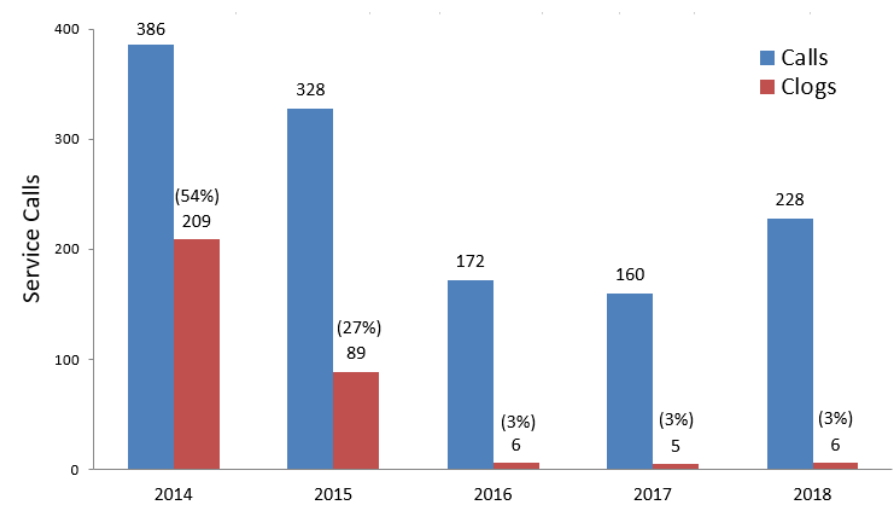

Figure 5: Analyses of service calls from 2014 to 2018 
Figure 6 provides an alternative perspective on the impact of the heterogeneous sample analysis method by depicting the increased robustness of the walk-up LCMS systems. The plot shows a 30-40 folds improvement in the performance of the instruments from 2016 to 2018, compared with 2014 which was used as the baseline for the comparison. In addition, the rinsing of the needle seat through the injection port has significantly reduced sample carry-over, which often proves to be an issue in a walkup environment. This has enabled us to shorten the needle washing step, reducing the injection cycle time by 30 seconds, which leads to an increase in throughput efficiency of $25 \%$ and $17 \%$ for the two- and three-minute methods respectively. This in turn allowed us to further improve operational efficiency via eliminating the requirement for replenishing wash vials manually with clean solvent.

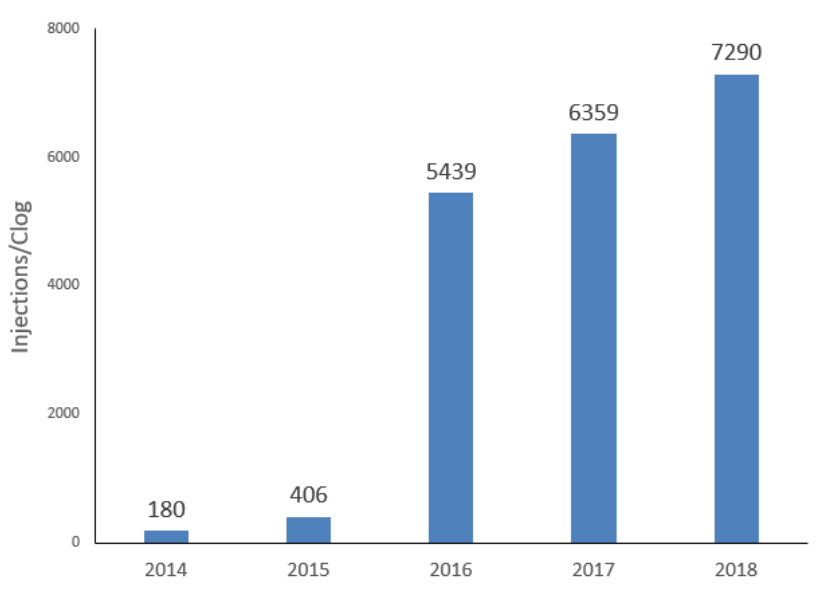

Figure 6: 30-Fold improvement in the robustness across six instruments since the implementation of the heterogeneous sample analysis method

\subsection{Reaction monitoring of heterogeneous samples}

With this simple solution in place, we have initiated efforts to implement routine reaction monitoring using one of our walk-up systems. One of the biggest drawbacks to achieving this goal in the past has been the inability to handle heterogeneous reactions. Although solutions to this have been developed utilizing ingenious sampling systems [11], the back-flush represents a simple method to mitigate this issue. Although, one can argue that utilizing this approach for monitoring heterogeneous reactions will not allow truly representative sampling of a reaction mixture, we have assembled a portfolio of examples, which we believe will provide value in a medicinal chemistry setting, and these examples will be reported in due course [12]

\section{Conclusion}

Herein, we have reported a simple and successful heterogeneous sample analysis method that we have implemented on six walk-up LCMS instruments which have been in operation for over 3 years. This method is easy to install and costs only a nominal amount per instrument to access the parts required. This has subsequently led to approximately a $95 \%$ reduction in the number of clogs. Decreased occurrences of clogging have increased the robustness and efficiency of our instruments while providing reliable chromatography for the users due to the elimination of 
pressure spikes. Most importantly, the system has resulted in significant savings in both consumables and time for both the chemists and instrument administrators.

\section{Acknowledgments}

The authors would like to thank Alex Yanovsky for informatics support; Vivian Thai for her assistance in early work; Bill Farrell, Jason Ewanicki, for helpful discussions, and Jennifer Lafontaine for management support.

\section{References}

1. Thomas, S.R.; Gerhard, U. J. Mass Spectrom. 2004, 39, 942 - 948, DOI : 10.1002/jms.673.

2. Pullen, F.S.; Perkins, G.L.; Burton, K.I; Ware, R.S; Teague, M.S; Kiplinger, J.P. J. Am. Mass Spectrom. 1995, 6, 394 - 399.

3. Mallis, L.M.; Sarkahian, A. B.; Kulishoff Jr., J.M.; Watts Jr., W.L. J. Mass Spectrom. 2002, 37, 889 - 896, DOI : 10.1002/jms.360.

4. A sample cleaning system has been disclosed in the patent literature. Zelechonok, Y.; Orlovsky, V. US 2010/0258487 (2009).

5. Svendsen, K.O.; Larsen, H.R.; Pedersen, S.A.; Brenna, I.; Lundanes, E.; Wilson, S.R. J. Sep. Sci. 2011, 34, 3020 - 3022, DOI : 10.1002/jssc.201100553.

6. Silva, M.R.; Brandtzaeg, O.K.; Vehus, T.; Lanças, F.M.; Wilson, S.R.; Lundanes, E. J. Chrom. A. 2017, 1518, 361 - 389, DOI : 10.1016/j.chroma.2017.08.071.

7. Johnsen, E.; Leknes, S.; Wilson, S.R.; Lundanes, E. Sci. Rep. 2015, 5, 9308, DOI : 10.1038/srep09308.

8. Van der Ven, H.C.; Gargano, A.F.G.; Van der Waal, S.J.; Schoenmakers, P.J. J. Chrom. A. 2016, 1427, 90 - 95, DOI : 10.1016/j.chroma.2015.11.082.

9. Wu, J.T.; Zeng, H.; Qian, M.; Brogdon, B.L.; Unger, S.E. Anal. Chem. 2000, 72, 61 - 67, DOI : 10.1021/ac990769y.

10. Roughley, S.D.; Jordan, A.M. J. Med. Chem. 2011, 54, 3451 - 3479, DOI : 10.1021/jm200187y.

11. Malig, T.C.; Koenig, J.D.B.; Situ, H.; Chehal, N.K.; Hultin, P.G.; Hein, J.E. React. Chem. Eng, 2017, 2, 309 - 314, DOI : 10.1039/c7re00026j.

12. Alimuddin, M.; Wang, W.; Richardson, P.; Braganza, J., Yanovsky, A. Manuscript in Preparation. 